\title{
HYPERCODES, RIGHT CONVEX LANGUAGES AND THEIR SYNTACTIC MONOIDS
}

\author{
G. THIERRIN ${ }^{1}$
}

\begin{abstract}
If $X^{*}$ is the free monoid generated by the alphabet $X$, then any subset $L$ of $X^{*}$ is called a language over $X$. If $P_{L}$ is the principal congruence determined by $L$, then the quotient monoid $\operatorname{syn}(L)=X^{*} / P_{L}$ is called the syntactic monoid of $L$. A hypercode over $X$ is any set of nonemtpy words that are noncomparable with respect to the embedding order of $X^{*}$. If $H$ is a hypercode, then the language $\tilde{H}=\left\{x \mid x \in X^{*}\right.$ and $a \leqslant x$ for some $\left.a \in H\right\}$ is a right convex ideal of $X^{*}$. The syntactic monoid $\operatorname{syn}(\tilde{H})$ can be characterized as a monoid with a disjunctive $\mu$-zero. The two particular interesting cases when $\operatorname{syn}(\tilde{H})$ is a nil monoid and when $\operatorname{syn}(\tilde{H})$ is a semillatice are also characterized.
\end{abstract}

1. Introduction and preliminary results. Let $X$ be an alphabet, finite or infinite, let $X^{*}$ be the free monoid generated by $X$ and let $X^{+}=X^{*}-\{1\}, 1$ being the empty word. The length of a word, $x \in X^{*}$, is denoted by $\lg (x)$ and every subset of $X^{*}$ is called a language over $X$.

If $M$ is a monoid and $A$ is a subset of $M$, then the relation $P_{A}$, defined by $a \equiv b\left(P_{A}\right)$ iff $A . . a=A . . b$ where $A . . a=\{(x, y) \mid x, y \in M, x a y \in A\}$ is a congruence of $M$, called the principal congruence determined by $A$. If $P_{A}$ is the identity, then $A$ is called disjunctive.

If $A$ is a language over $X$, then $P_{A}$ is called the syntactic congruence of $A$ and the quotient monoid $\operatorname{syn}(A)=X^{*} / P_{A}$ is called the syntactic monoid of $A$.

The relation $\leqslant$ defined on $X^{*}$ by $x \leqslant y$ iff $x=x_{1} x_{2} \cdots x_{n}$ and $y=$ $y_{1} x_{1} y_{2} x_{2} \cdots y_{n} x_{n} y_{n+1}$ for some $n$ where $x_{i}, y_{i} \in X^{*}$ is a partial order on $X^{*}$, called the embedding order. For every language $A \subseteq X^{*}$, let $\tilde{A}=\{x \mid a<x$ for some $a \in A\}$ and $\underset{\sim}{A}=\{x \mid x \leqslant a$ for some $a \in A\}$. It is well known that if $X$ is finite, then each set of pairwise incomparable elements of $X^{*}$ is always finite ([1], [2], [3]), and that the languages $\tilde{A}$ and $\boldsymbol{A}$ are regular, that is, their syntactic monoids are finite.

A nonempty language, $C \subseteq X^{+}$, is called a code if $a_{1} a_{2} \cdots a_{n}=b_{1} b_{2} \cdots b_{m}$ and $a_{i}, b_{j} \in C$ implies $n=m$ and $a_{i}=b_{i}$ for every $i$. A nonempty language $H \subseteq X^{+}$is called a hypercode if every pair of distinct elements of $H$ are incomparable relative to the embedding order. It is immediate that every hypercode is a code.

Received by the editors June 28, 1980 and, in revised form, January 13, 1981.

1980 Mathematics Subject Classification. Primary 20M35; Secondary 68A30.

Key words and phrases. Monoid, language, syntactic monoid, embedding order, hypercode,،convex, nil monoid, semilattice.

${ }^{1}$ This research has been supported by Grant A7877 of the Natural Sciences and Engineering Research Council of Canada. 
A language $A \subseteq X^{*}$ is said to be right convex if $a \leqslant x, a \in A$ implies $x \in A$. A language $A, 1 \notin A$, is right convex iff $A=\tilde{H}$ for some hypercode $H$. This hypercode $H$ is the set of the minimal words of $A$. Right convex languages and hypercodes have been first considered when the alphabet $X$ is finite ([7], [8]). In this case, the hypercodes are always finite and the right convex languages are regular.

An ideal $I$ of a monoid $M$ is called a $\mu$-ideal if $a b \in I$ implies $a x b \in I$ for all $x \in M[10]$ and a zero element of $M$ is called a $\mu$-zero if it is a $\mu$-ideal. A language $A$ over $X$ is right convex iff $A$ is a $\mu$-ideal.

If $H$ is a hypercode over $X$, then $\operatorname{syn}(\tilde{H})$ is a monoid with a disjunctive $\mu$-zero. Conversely, if $M$ is a monoid with a disjunctive $\mu$-zero, then there exists a hypercode over an alphabet $X$ such that $M$ is isomorphic to $\operatorname{syn}(\tilde{H})$.

Some properties of a language can be determined by considering their syntactic monoid. For example, a language $A$ over a finite alphabet is regular iff $\operatorname{syn}(A)$ is finite. If the language $C$ is a code, then it is in general more interesting to consider the syntactic monoid $\operatorname{syn}\left(C^{*}\right)$ of $C^{*}$ instead of $C$. However, this is not the case for the hypercodes. A characterization of the syntactic monoid $\operatorname{syn}(H)$ of a hypercode $H$ over a finite alphabet has been given in [9]. In this paper, we consider the syntactic monoid of languages of the form $\tilde{H}$ where $H$ is a hypercode. In particular, we characterize the hypercodes $H$ in the two following cases:

(a) $\operatorname{syn}(\tilde{H})$ is a nil monoid and the alphabet $X$ is finite;

(b) $\operatorname{syn}(\tilde{H})$ is a semilattice.

2. Quasi-maximal hypercodes over a finite alphabet. In this section, the alphabet $X$ is always assumed to be finite. A hypercode $H$ over $X$ is said to be maximal if for every, $u \in X^{*}, u \notin H, H \cup u$ is not a hypercode. Every hypercode can be embedded in a maximal one. A hypercode is maximal iff $X^{*}=\tilde{H} \cup \underset{\sim}{H}$ [7]. A hypercode is said to be quasi-maximal if $X^{*}-\{\tilde{H} \cup \underset{\sim}{H}\}$ is finite. Since the alphabet $X$ is finite, and since a hypercode over $X$ is always finite, then $\underset{\sim}{H}$ is also finite; therefore, $H$ is a quasi-maximal hypercode iff $X^{*}-\tilde{H}$ is finite. Clearly, every maximal hypercode is quasi-maximal, but the converse is not true. For example, if $X=\{a, b\}$, then $H=\left\{a^{2}, b^{2}\right\}$ is a hypercode that is quasi-maximal but not maximal.

Let us remark that a hypercode $H$ over a finite alphabet $X$ is quasi-maximal iff there exists an integer $m \geqslant 1$ such that $H \cup u$ is not a hypercode for $\lg (u)>m$.

Recall that a nil monoid is a monoid with zero such that every element different from the identity is nilpotent.

If $A$ is a language over $X$ we will denote by $\alpha(A)$ the alphabet of $A$, that is, $\alpha(A)=\left\{a \mid a \in X\right.$ and $r a s \in A$ for some $\left.r, s \in X^{*}\right\}$.

Proposition 1. Let $H$ be a hypercode over a finite alphabet $X$ such that $\alpha(H)=$ $X$. Then $H$ is quasi-maximal $\Leftrightarrow \operatorname{syn}(\tilde{H})$ is a finite nil monoid.

Proof. $(\Rightarrow)$ Let $u \in X^{*}, u \neq 1$, and suppose that $u^{m} \notin \tilde{H}$ for $m>1$. Since $H$ is quasi-maximal, there exists $k \geqslant 1$ such that $H \cup u^{n}$ is not a hypercode for $n>k$. Hence $u^{n} \in \underset{\sim}{H}$ for $n>k$. Since $\underset{\sim}{H}$ is finite, we have a contradiction. Therefore, 
$u^{m} \in \tilde{H}$ for some $m \geqslant 1$ and $\operatorname{syn}(\tilde{H})$ is a nil monoid because the class $\tilde{H}$ modulo $P_{\tilde{H}}$ is the zero element of $\operatorname{syn}(\tilde{H})$. Since $H$ is finite, then $\tilde{H}$ is regular and $\operatorname{syn}(\tilde{H})$ is finite.

$(\Leftrightarrow)$ Let $a \in X$. Since $\alpha(H)=X$, there exist $r, s \in X^{*}$ such that $h_{1}=r a s \in H$. If $a \equiv 1\left(P_{\tilde{H}}\right)$, then $h_{1}=$ ras $\equiv r s\left(P_{\tilde{H}}\right)$. Since $\tilde{H}$ is a class modulo $P_{\tilde{H}}$, then $r s \in \tilde{H}$ and $h_{2}<r s$ for some $h_{2} \in H$. Therefore, $h_{2}<r s<h_{1}$ and $h_{2}=r s=h_{1}$, a contradiction. Hence $a \neq 1\left(P_{\tilde{H}}\right)$.

Since $\operatorname{syn}(\tilde{H})$ is a finite nil monoid, then for every $a \in X$, there exists $m>1$ such that $a^{m} \in \tilde{H}$. Suppose that $X^{*}-\{\tilde{H} \cup \underset{\sim}{H}\}=K$ is infinite. Then there exists $w \in K$ containing at least $m$ identical letters of the alphabet $X$, say $a$. Therefore, $w=x_{1} a x_{2} a \cdots a x_{m} a x_{m+1}$ where $x_{i} \in X^{*}$. Since $a^{m} \in \tilde{H}$, then $a^{m}<w$ and $w \in$ $\tilde{H}$, a contradiction. It follows then that $H$ is quasi-maximal.

Remark that if $H$ is a quasi-maximal hypercode over a finite alphabet, then $\operatorname{syn}(\tilde{H})$ is a nil monoid with a disjunctive $\mu$-zero and $\operatorname{syn}(\tilde{H})$ is subdirectly irreducible, because every nil monoid with a disjunctive zero is subdirectly irreducible [6].

3. Hypercodes and semilattices with disjunctive zero. In this section, we characterize the hypercodes $H$ such that $\operatorname{syn}(\tilde{H})$ is a semilattice with a disjunctive zero. Let us remark that if a semilattice has a zero, then the zero element is disjunctive iff the sets of the zero divisors of two distinct elements are always distinct.

If $B(\vee, \wedge)$ is a boolean algebra, then the zero element 0 of $B$ (the identity element 1 of $B$ ) is a disjunctive zero relative to the operation $\wedge$ (the operation $\vee$ ). A semilattice or a lattice with a disjunctive zero is not in general a boolean algebra. For example, let $L=\left\{0,1, a_{1}, a_{2}, a_{3}\right\}$ with $a_{i} \wedge a_{j}=0$ and $a_{i} \vee a_{j}=1$ for $i \neq j$. Clearly, 0 is a disjunctive element for the operation $\wedge$, but $L$ is not a boolean algebra. Conditions for a subset, and, in particular, for an element of a semilattice to be disjunctive can be found in [4].

Recall that an ideal $A$ of a monoid $M$ is said to be cs-prime (or completely semiprime) if $x^{n} \in A, n$ a positive integer, implies $x \in A$. Remark that an ideal $A$ of a monoid $M$ is cs-prime iff the quotient monoid $M / P_{A}$ is a semilattice with a disjunctive zero.

Let $A$ be a language over $X . A$ is said to be power free if $x a^{n} y \in A$ with $a \neq 1$, $x, y \in X^{*}, n \geqslant 1$, implies $n=1$. $A$ is said to be completely reflective if $u v w \in H$ with $u, v, w \in X^{*}$ implies wvu $\in H$.

Proposition 2. Let $\mathrm{H}$ be a hypercode. Then the following properties are equivalent.

(1) $\tilde{H}$ is cs-prime.

(2) $H$ is power free and completely reflective.

(3) $\operatorname{syn}(\tilde{H})$ is a semilattice with disjunctive zero.

(4) $\operatorname{syn}(\tilde{H})$ is a semilattice.

Proof. (1) $\Rightarrow$ (2). Suppose that $u v^{2} w \in H, v \neq 1$. Then $u v \cdot w u \cdot v w \in \tilde{H},(u v w)^{2}$ $\in \tilde{H}$ and $u v w \in \tilde{H}$. Hence $h \leqslant u v w$ for some $h \in H$. From $h \leqslant u v w \leqslant u v^{2} w$ it follows that $h=u v w=u v^{2} w$, a contradiction. Therefore, $H$ is power free. 
Let $m$ be the length of the words of minimal length in $H$ and let $u v w \in H$ with $\lg (u v w)=m$. Then $w v u \in \tilde{H}$ because, by the above remark, $\operatorname{syn}(\tilde{H})$ is commutative. Hence, $h<w v u$ for some $h \in H$, and, therefore, $h=w v u \in H$. Suppose now that for all words $u v w$ of length $\lg (u v w)<n$, $u v w \in H$ implies $w v u \in H$. Let $u v w \in H$ with $\lg (u v w)=k>n$ such that for every $r \in H$ with $\lg (r)<k$ we have $\lg (r) \leqslant n$. Since $\operatorname{syn}(\tilde{H})$ is commutative, then $w v u \in \tilde{H}$ and there exists $h \in H$ such that $h \leqslant w v u$. Suppose that wvu $\notin H$. Then $\lg (h)<\lg (w v u)$ and $\lg (h)<n$. Furthermore, $h=w_{1} v_{1} u_{1}$ with $w_{1} \leqslant w, v_{1} \leqslant v, u_{1}<u$. Since $\lg (h)<n$, then $u_{1} v_{1} w_{1}$ $\in H$. But $u_{1} v_{1} w_{1} \leqslant u v w$, and, therefore, $u_{1} v_{1} w_{1}=u v w$, a contradiction. Hence, $w v u \in H$ and $H$ is completely reflective.

(2) $\Rightarrow(1)$. Since $\tilde{H}$ is an ideal, in order to show that $\tilde{H}$ is cs-prime, it is sufficient to show that $u^{2} \in \tilde{H}$ implies $u \in H$. Suppose that $u \notin \tilde{H}$. Then there exists $v \in H$ such that $v \leqslant u^{2}$ and $v \nless u$. Let $v=v_{1} v_{2} \cdots v_{m}$ with $v_{i} \in X$. Since $H$ is completely reflective, then $v_{i_{1}} v_{i_{2}} \cdots v_{i_{m}} \in H$ for every permutation $i_{1} i_{2} \cdots i_{m}$ of $1,2, \ldots, m$. Since $H$ is power free, then $v_{i} \neq v_{j}$ for $i \neq j$. From $v<u^{2}$, we have $v=v_{1} \cdots v_{r} v_{r+1} \cdots v_{m}$ with $v_{1} v_{2} \cdots v_{r} \leqslant u$ and $v_{r+1} \cdots v_{m}<u$. It follows then that $u=x_{1} v_{i_{1}} x_{2} v_{i_{2}} \cdots x_{m} v_{i_{m}} x_{m+1}$ with $x_{j} \in X^{*}$ and $v^{\prime}=v_{i_{1}} v_{i_{2}} \cdots v_{i_{m}}$ is obtained from $v$ by a permutation of its letters. But $v^{\prime} \in H$. Therefore, $v^{\prime}<u$ and $u \in \tilde{H}$, a contradiction.

(1) $\Leftrightarrow$ (3). Immediate.

(3) $\Rightarrow$ (4). Trivial.

(4) $\Rightarrow(3) . \tilde{H}$ is a class modulo $P_{\tilde{H}}$, and since $\tilde{H}$ is an ideal, then $\tilde{H}$ is a disjunctive zero of $\operatorname{syn}(\tilde{H})$.

Remark that in Proposition 2 the semilattice $\operatorname{syn}(\tilde{H})$ always has an identity element. It is immediate that if $S$ is a semilattice with a disjunctive zero and an identity element, then $S$ is isomorphic to $\operatorname{syn}(\tilde{H})$, where $H$ is a power free and completely reflective hypercode $H$ over some alphabet $X$.

\section{REFERENCES}

1. L. H. Haines, On free monoids partially ordered by embedding, J. Combin. Theory 6 (1969), 94-98.

2. G. Higman, Ordering by divisibility in abstract algebras, Proc. London Math. Soc. 2 (1952), 225-336.

3. M. P. Jullien, Sur un théorème d'extension dans la théorie des mots, C. R. Acad. Sci. Paris Sér. A 266 (1968), 651-654.

4. H. Jürgensen, Inf-halbverbände als syntaktische Halbgruppen, Acta Math. Acad. Sci. Hungar. 31 (1978), 37-41.

5. G. Lallement, Semigroups and combinatorial applications, Wiley, New York, 1978.

6. B. M. Schein, Homomorphisms and subdirect decompositions of semigroups, Pacific J. Math. 17 (1966), 529-547.

7. H. J. Shyr and G. Thierrin, Hypercodes, Inform. and Control 24 (1974), 45-54.

8. G. Thierrin, Convex languages, Proc. IRIA Sympos. Automata, Languages and Programming (Paris, 1972), North-Holland, Amsterdam, 1972, pp. 481-492.

9.

10. Decomposition of some classes of subsets in a semigroup, Proc. Conf. Semigroups and Applications (Oberwolfach, 1978), Springer-Verlag, Berlin and New York, 1981.

Department of Mathematics, University of Western Ontario, London, Ontario N6a 5B9, CANADA 